\title{
Triatoma tibiamaculata (Pinto, 1926): Tábua de vida das ninfas, duração das formas adultas e postura das fêmeas (Hemiptera - Reduviidae)
}

\author{
Triatoma tibiamaculata (Pinto, 1926): life table for nymphs, \\ duration of adult forms and oviposition of females
}

\author{
Vera Lúcia Cortiço Corrêa Rodrigues ${ }^{1}$, Antenor Nascimento Ferraz Filho ${ }^{1}$ \\ e Eduardo Olavo da Rocha e Silva ${ }^{1}$
}

\begin{abstract}
RESUMO
Os autores analisaram em condições de laboratório, a taxa de sobrevida das ninfas, duração mínima e máxima de cada estádio, tempo de vida das formas adultas e postura das fêmeas. Foram acompanhados dois grupos de 100 ovos. Lote A, exemplares criados em um único cristalizador. Lote B os exemplares foram mantidos isolados um a um e ao atingir a fase alada formaram 20 casais, possibilitando o controle da postura das fêmeas e o tempo de vida de cada exemplar. O percentual de eclosão dos ovos foi de 96\%; a taxa de vida no final da fase ninfal foi de 69,5\% no Lote A e de 78,4\% no Lote B. A maior freqüência observada no tempo decorrido entre postura e eclosão da ninfa do $1^{\circ}$ estádio foi de 28 dias. $O$ tempo de permanência na fase de ninfa foi de 4 a 8 meses e de 5 meses na fase adulta. A postura total (média) no Lote $B$ foi de 181,6 ovos por fêmea.
\end{abstract}

Palavras-chaves: Triatoma tibiamaculata. Triatominae. Tábua de vida. Doença de Chagas.

\begin{abstract}
Under laboratory conditions, the authors analyzed the survival rate of nymphs, the minimum and maximum duration of each stage, the length of life of the winged forms and oviposition of the females. Two groups of 100 eggs each were monitored. In batch A, the specimens were reared in a single glass receptacle. In batch B, the specimens were kept apart, one by one, and when they reached the winged stage, they formed twenty couples, which made it possible to watch over the oviposition of the females and the length of life of each specimen. The egg eclosion rate was 96\%; the proportion still alive at the end of the nymph phase was $69.5 \%$ in batch A and $78.4 \%$ in batch B. The most frequent length of time observed between oviposition and eclosion of first-stage nymphs was 28 days. The nymph phase lasted four to eight months and the adult phase five months. The mean total oviposition in batch B was 181.6 eggs per female.
\end{abstract}

Key words: Triatoma tibiamaculata. Triatominae. Life table. Chagas' disease.

As alterações provocadas pela ação predatória do homem sobre a cobertura florestal nativa presente na região litorânea do Estado de São Paulo, acabaram por transformar a invasão ocasional das moradias pelos triatomíneos, em um fato já que chamava a atenção de Forattini e cols ${ }^{4}$ em 1978. Entre as espécies que assim procedem, encontra-se o Triatoma tibiamaculata, cuja área de dispersão atinge não apenas a Mata Atlântica em São Paulo, como também nos Estados do Paraná e Santa Catarina, mais ao Sul e Rio de Janeiro, Espírito Santo, Minas Gerais, Bahia e Sergipe ao norte ${ }^{913}$. Esta espécie é rotulada como silvestre pela inexistência de registros que demonstrem sua capacidade de formar, espontaneamente, colônias nos ecótopos artificiais. Quando suas formas aladas são encontradas nas habitações, são consideradas visitantes ${ }^{12}$. Por outro lado o interesse pela espécie aumentou em nosso meio, pelo encontro de exemplares com infecção natural ${ }^{2}$ e de casos humanos da doença de Chagas autóctones em municípios, localizados no litoral sul paulista ${ }^{15611}$. Os escassos relatos na literatura especializada sobre o Triatoma tibiamaculata, notadamente quanto a aspectos da biologia, foi dos fatores que nos levaram a efetuar estudos, em condições do

\footnotetext{
1.Superintendência de Controle de Endemias, Mogi Guaçu, SP.

Endereço para correspondência: Drª Vera Lúcia Cortiço Corrêa Rodrigues. SUCEN. Rua Afonso Pessini 86, Caixa Postal 192, 13840-970 Mogi Guaçu, SP. Tel: 55 193861-1233

e-mail: veracorrea@dglnet.com.br

Recebido em: 01/08/2006

Aceito em: 20/06/2007
} 
laboratório, preliminarmente a respeito do tempo de vida dos imagos e postura das fêmeas ${ }^{3}$, completado com observações a respeito da taxa de sobrevida das ninfas e duração de cada estádio.

\section{MATERIAL E MÉTODOS}

Inicialmente, acompanhamos a postura de 10 fêmeas do Triatoma tibiamaculata, cuja produção em sete dias consecutivos atingiu 200 ovos. Oriundas do insetário da SUCEN, situado em Mogi Guaçu, SP essas fêmeas representavam parte da primeira filiação de um exemplar adulto, capturado na zona rural do município de Iguape, SP. Logo após a postura (oitavo dia), os ovos foram aleatoriamente distribuídos em dois lotes iguais, contendo cada um 100 ovos. Do Lote A, eclodiram ninfas que durante $o$ transcurso da observação permaneceram abrigadas em um cristalizador de acrílico, fumê, com cerca de $25 \mathrm{~cm}$ de diâmetro e $10 \mathrm{~cm}$ de altura. Em decorrência, se propiciou a formação de uma colônia única que de ovos, evoluiu a adultos. Do Lote B, eclodiram ninfas do primeiro estádio que independentemente do número de exemplares, diariamente, eram transferidas para caixas plásticas semitransparentes, com $6 \mathrm{~cm}$ de diâmetro e $5 \mathrm{~cm}$ de altura. $\mathrm{Na}$ medida em que iam acontecendo as ecdises subseqüentes, as caixas que continham mais de uma ninfa foram diminuindo de número, até que ao atingir o quinto estádio, todas as ninfas já estavam isoladas em caixa plástica própria. Deste modo, foi possível ao atingir a fase de imago, se compor 20 casais jovens mantidos segregados entre si, fato que permitiu a anotação do tempo de vida e postura de cada exemplar fêmea. Ao final do trabalho, foi comparada a postura total de cada lote. No que se refere às ninfas, foi possível apenas registrar os tempos, mínimo e máximo, de duração de cada estádio e respectivas taxas de sobrevida.

Os dois lotes permaneceram durante toda a observação em uma das dependências do insetário de Mogi Guaçu, onde a temperatura foi mantida a $30^{\circ} \mathrm{C}$ e a umidade relativa do ar em $80 \%$. Os exemplares eram alimentados semanalmente, em repasto com uma hora de duração, realizados em aves: galos no Lote A e pombos no Lote B. As anotações necessárias eram efetuadas todos os dias, inclusive sábados e domingos.

Os resultados são descritos sob a forma de médias aritméticas, medianas e taxas de sobrevida. As definições e os parâmetros estatísticos mencionados são os utilizados por Rabinovich ${ }^{10}$.

\section{RESULTADOS}

As Tabelas 1 e 2 apresentam as tábuas de vida do Triatoma tibiamaculata, na fase de ninfa. A Tabelas 1 mostra a taxa de sobrevida observada entre as ninfas criadas em conjunto, ou seja, na forma de colônia única Lote A. A Tabela 2 mostra a mesma taxa obtida entre as ninfas mantidas segregadas nas caixas plásticas (Lote B). Em virtude dos diferentes procedimentos adotados quanto aos processos de criação e alimentação, esses resultados não são comparáveis. Em ambos os lotes, a mortalidade foi significativa, o que proporcionou uma taxa de sobrevida final de $69,5 \%$ no Lote A, e uma taxa de sobrevida de $78,4 \%$ no Lote B. A maior mortalidade aconteceu no decorrer do segundo estádio, sendo de 10 mortes no Lote A e de 13 mortes no Lote B.
Tabela 1 - Triatoma tibiamaculata: tábua de sobrevida das ninfas criadas em conjunto e alimentadas em aves (Lote A). SUCEN/Insetário de Mogi Guaçu.

\begin{tabular}{|c|c|c|c|c|c|}
\hline Fase do ciclo evolutivo & $\begin{array}{c}1_{\mathrm{n}} \\
(100 \text { ovos })\end{array}$ & $\mathrm{d}_{\mathrm{n}}$ & $q_{n}$ & $\mathrm{p}_{\mathrm{n}}$ & $P_{n}(\%)$ \\
\hline $1^{0}$ estágio & 95 & 7 & 0,0737 & 0,9263 & 92,6 \\
\hline $2^{\circ}$ estágio & 88 & 10 & 0,1136 & 0,8864 & 82,1 \\
\hline $3^{0}$ estágio & 78 & 4 & 0,0513 & 0,9487 & 77,9 \\
\hline $4^{0}$ estágio & 74 & 3 & 0,0405 & 0,9595 & 74,7 \\
\hline 5ำ estágio & 71 & 5 & 0,0704 & 0,9296 & 69,5 \\
\hline Alado & 66 & 29 & & & \\
\hline QQ & & & & & \\
\hline 35 & & & & & \\
\hline \multicolumn{2}{|c|}{$\begin{aligned} \text { Legenda: } 1_{\mathrm{n}}=\text { vivos no início da fase } \\
$\[ \mathrm{q}_{\mathrm{n}}=\frac{{ }_{\mathrm{n}}}{{ }_{{ }_{\mathrm{n}}}} \quad \mathrm{p}_{\mathrm{n}}=1-\mathrm{q}_{\mathrm{n}} \]$\end{aligned}$} & \multicolumn{4}{|c|}{$\begin{array}{c}d_{n}=\text { mortos no decorrer da fase } \\
P_{n}=\left(p_{n} \times p_{n}-1\right) \%\end{array}$} \\
\hline
\end{tabular}

Tabela 2 - Triatoma tibiamaculata: tábua de sobrevida das ninfas criadas em isoladas e alimentadas em pombos (Lote B). SUCEN/Insetário de Mogi Guaçu.

\begin{tabular}{|c|c|c|c|c|c|}
\hline Fase do ciclo evolutivo & $\begin{array}{c}1_{\mathrm{n}} \\
(100 \text { ovos })\end{array}$ & $d_{n}$ & $q_{n}$ & $\mathrm{p}_{\mathrm{n}}$ & $P_{n}(\%)$ \\
\hline $1^{\circ}$ estágio & 97 & 4 & 0,0412 & 0,9588 & 95,9 \\
\hline $2^{\circ}$ estágio & 93 & 13 & 0,1398 & 0,8602 & 82,5 \\
\hline $3^{\circ}$ estágio & 80 & 3 & 0,0375 & 0,9625 & 79,4 \\
\hline $4^{\circ}$ estágio & 77 & 1 & 0,0130 & 0,9870 & 78,4 \\
\hline $5^{\circ}$ estágio & 76 & 21 & 0,0000 & 1,000 & 78,4 \\
\hline Alado & 66 & 29 & & & \\
\hline$Q Q$ & & & & & \\
\hline 37 & & & & & \\
\hline $\begin{array}{r}\text { Legenda: } \begin{aligned} 1_{n} & =\text { vivos no } \\
q_{n}=\frac{{ }_{n}}{1_{n}} & p_{1}\end{aligned}\end{array}$ & $\begin{array}{l}\text { io da fase } \\
1-q_{n}\end{array}$ & & $\begin{array}{l}\mathrm{d}_{\mathrm{n}}=\operatorname{mortc} \\
\mathrm{P}_{\mathrm{n}}=\left(\mathrm{p}_{\mathrm{n}}\right.\end{array}$ & $\begin{array}{l}\text { no decor } \\
\left.x p_{n}-1\right)\end{array}$ & rer da fase \\
\hline
\end{tabular}

A Tabela 3 apresenta o tempo de duração de cada fase do ciclo de vida dos triatomíneos no Lote B. Vinte e oito dias foi a maior frequiência observada no intervalo de tempo decorrido entre a postura do ovo e eclosão da respectiva ninfa. A seguir são apresentados na mesma tabela, o tempo mínimo e máximo de duração das ninfas de $5^{\circ}$ estádio e das formas adultas. 0 período total da fase de ninfa ficou entre quatro e oito meses. No caso das formas adultas, o tempo médio de vida foi de 156 dias, para os machos e 167 dias para as fêmeas.

A Tabela 4 mostra a postura total (ovos férteis e inférteis) das fêmeas acasaladas com um só macho (Lote B). Nela, observamos que a menor postura foi de 31 ovos (casal 11) e a maior de 381 ovos (casal 18), sendo de 181,6 ovos a postura total média das vinte fêmeas consideradas e de 1,1 a postura-dia, por fêmea.

Ao finalizar, comparamos as posturas totais alcançadas em cada lote onde podemos observar que o maior número de ovos férteis é conseguido quando as fêmeas são mantidas em colônia (Tabela 5). 
Tabela 3 - Triatoma tibiamaculata: duração mínima e máxima da fase de ninfa no (Lote B). SUCEN/Insetário de Mogi Guaçu

\begin{tabular}{|c|c|c|c|c|}
\hline \multirow{5}{*}{$\begin{array}{l}\text { Fase do ciclo evolutivo } \\
\text { da postura do ovo à } \\
\text { eclosão da ninfa } \\
\text { Fase de ninfa (dias) }\end{array}$} & \multicolumn{4}{|c|}{ Tempo de duração (em dias) } \\
\hline & \multicolumn{2}{|c|}{ menor } & \multicolumn{2}{|c|}{ maior } \\
\hline & \multicolumn{2}{|c|}{ período observado } & \multicolumn{2}{|c|}{ período observado } \\
\hline & \multicolumn{4}{|c|}{28 dias* } \\
\hline & Macho & Fêmea & Macho & Fêmea \\
\hline $1^{0}$ estágio & 10 & 13 & 31 & 40 \\
\hline $2^{2}$ estágio & 14 & 14 & 31 & 29 \\
\hline 3ํestágio & 12 & 12 & 41 & 42 \\
\hline $4^{0}$ estágio & 13 & 12 & 97 & 96 \\
\hline 5ํestágio & 56 & 40 & 122 & 112 \\
\hline \multicolumn{5}{|l|}{ Período total (dias) } \\
\hline fase ninfa & 151 & 136 & 248 & 240 \\
\hline fase adulta** & 91 & 77 & 277 & 238 \\
\hline
\end{tabular}

Tabela 4 - Triatoma tibiamaculata: postura total das fêmeas acasaladas com um único adulto macho; no período de 23 dias (Lote B). SUCEN/ Insetário de Mogi Guaçu.

\begin{tabular}{|c|c|c|c|}
\hline Casal n ${ }^{\circ}$ & Total de ovos & Ovos férteis & Ovos não férteis \\
\hline 01 & 115 & 74 & 41 \\
\hline 02 & 373 & 321 & 52 \\
\hline 03 & 58 & 37 & 21 \\
\hline 04 & 243 & 182 & 61 \\
\hline 05 & 104 & 34 & 70 \\
\hline 06 & 157 & 75 & 82 \\
\hline 07 & 147 & 86 & 61 \\
\hline 08 & 163 & 142 & 21 \\
\hline 09 & 106 & 82 & 24 \\
\hline 10 & 121 & 80 & 41 \\
\hline 11 & 31 & 12 & 19 \\
\hline 12 & 191 & 52 & 139 \\
\hline 13 & 248 & 211 & 37 \\
\hline 14 & 205 & 106 & 99 \\
\hline 15 & 282 & 194 & 88 \\
\hline 16 & 212 & 166 & 46 \\
\hline 17 & 78 & 59 & 19 \\
\hline 18 & 381 & 286 & 95 \\
\hline 19 & 225 & 167 & 58 \\
\hline 20 & 193 & 101 & 92 \\
\hline Total geral & 3.633 & 2.467 & 1.166 \\
\hline média & 181,6 & 123,3 & 58,3 \\
\hline mediana & 177,0 & 93,5 & 55,0 \\
\hline
\end{tabular}

Tabela 5 - Triatoma tibiamaculata: condições dos ovos nas posturas das fêmeas. Casais em colônias ou em frascos separados. SUCEN/Insetário de Mogi Guaçu.

Casais de Triatoma tibiamaculada

\begin{tabular}{lcrrrr}
\cline { 2 - 3 } Postura & \multicolumn{2}{c}{ mantidos em colônia } & & \multicolumn{2}{c}{ criados em frascos separados } \\
\cline { 2 - 3 } \cline { 5 - 6 } & $\mathrm{n}^{\underline{0}}$ & $\%$ & & $\mathrm{n}^{0}$ & $\%$ \\
\hline Ovos férteis & 4.617 & 58,9 & & 2.467 & 67,9 \\
Ovos inférteis & 3.215 & 41,1 & & 1.166 & 32,1 \\
\hline Total (ovos) & 7.832 & 100,0 & & 3.633 & 100,0 \\
\hline
\end{tabular}

\section{DISCUSSÃo}

0 percentual de eclosão dos ovos que iniciaram as colônias e que deram origem aos Lotes A e B foi de $96 \%$. A mortalidade das ninfas foi mais acentuada no Lote A (colônia única). A maior mortalidade aconteceu no $2^{\circ}$ estádio sendo 10 mortes no Lote A e 13 mortes no Lote B. Resultado diferente foi encontrado por Galvão ${ }^{7}$ com o Triatoma nitida e Rabinovich ${ }^{10}$ com o Triatoma infestans que observaram uma maior mortalidade das ninfas de $5^{\circ}$ estádio. Triatoma tibiamaculata apresentou um período maior de duração na fase de ninfa, de 248 dias para os exemplares machos e 238 dias para os exemplares fêmeas, semelhante ao encontrado por Souza cols ${ }^{14}$, em 1978, com o Triatoma sordida que observaram para a fase de ninfa dos exemplares machos o período de 249 dias e para as fêmeas um período um pouco maior 259 dias. 0 período médio de vida das adultas foi de 156 dias para os machos e 167 dias para as fêmeas tempo bem menor do que o encontrado por Souza ${ }^{14}$, para o Triatoma sordida, que foi de 284 dias e 503 dias para macho e fêmea, respectivamente. Um dos períodos mais longos já relatados na literatura foi o encontrado por Galvão ${ }^{7}$, com o Triatoma nitida que apresentou uma média de duração do ciclo, da eclosão à fase adulta de 897,6 dias. Souza ${ }^{14}$ observou um período médio para as fêmeas de Triatoma sordida de 673,6 dias. As taxas de sobrevivência, ao atingirem a fase alada, foram $69,5 \%$ no Lote A (colônia única) e de 78,4\% no Lote B (exemplares isolados), talvez isto se deva ao maior manuseio da colônia única. A postura total foi maior na colônia única (Lote A) 7.832 ovos, a percentagem de ovos inférteis também foi no maior no Lote A. A maior quantidade de ovos postos por uma fêmea foi de 381 ovos diferente do Triatoma sordida ${ }^{14}$ em que as fêmeas mostraram-se como verdadeiras poedeiras e uma das fêmeas observadas colocou 1.281 no período em que foi acompanhada. O Lote B (exemplares isolados) com a vantagem da criação individual permitiu uma observação detalhada do tempo de vida e postura das fêmeas.

0 tempo de vida (aproximado) dos exemplares que atingiram a fase adulta alcançou 12 meses.

\section{AGRADECIMENTO}

Os autores agradecem aos funcionários Pedro Ribeiro da Silva e Vera Lúcia Braga Tonietti, pelo suporte técnico.

\section{REFERÊNCIAS}

1. Ciaravolo RM, Domingos MF, Wanderley DMV, Gerbi JL, Chieffi PP, Peres AB, Umezawa ES. Doença de Chagas aguda no Estado de São Paulo: aspectos epidemiológicos. Revista do Instituto de Medicina Tropical de São Paulo 39:171-174, 1997.

2. Dias-Lima AG, Sherlock IA. Sylvatic vectors invading houses and the risk of emergence of cases of Chagas disease in Salvador, State of Bahia, Northeast Brazil. Memórias do Instituto Oswaldo Cruz 95:611-613, 2000.

3. Ferraz Filho AN, Silva EOR. Tempo de vida das formas aladas e postura das fêmeas do Triatoma tibiamaculata. In: Resumos do XIX Congresso da Sociedade Brasileira de Medicina Tropical p. 46, 1983. 
4. Forattini OP, Ferreira OA, Silva EOR, Rabello EX. Aspectos ecológicos da Tripanossomíase Americana. XII Variação regional da tendência de Panstrongylus megistus à domiciliação. Revista de Saúde Pública 12:209-233, 1978.

5. Forattini OP, Silva EOR, Barata JMS, Boainain. Nota sobre caso autóctone de Tripanossomíase Americana no litoral sul do Estado de São Paulo, Brasil. Revista Saúde Pública 14:143-149, 1980.

6. Forattini OP, Silva EOR, Barata JMS, Boainain E. Nota sobre novo caso autóctone de Tripanossomíase Americana no litoral sul do Estado de São Paulo, Brasil. Revista Saúde Pública 15:350-352,1981.

7. Galvão C, Juberg J, Cunha V, Mello RP. Biologia do Triatoma nitida Usinger, 1939 em laboratório (Hemiptera:Reduviidae). Memórias do Instituto Oswaldo Cruz 90:657-663, 1995.

8. Lent H, Wygodzinsky P. Revision of the Triatominae (Hemiptera: Reduviidae), and their significance as vectors of Chagas' Disease. Bulletin of the American Museum of Natural History 163: 125-520, 1979.

9. Miles MA. Distribution and Importance of Triatominae as vectors of T. cruzi. In: Abstract of International Symposium on New Approaches in American
Trypanosomiasis Research Belo Horizonte MG. Brasil, 1975. Proceedings (PAHO), Scientific Publication nr 318, Pan American Health Organization, Washington p. 48-53, 1976.

10. Rabinovich JE. Vital statistics of Triatominae (Hemiptera: Reduviidae) under laboratory conditions. I. Triatoma infestans Klug. Journal Medicina of Entomology 9: 351-370, 1972.

11. Rodrigues VLCC, Condino MLF, Santos FC, Silva RA, Baitelo D. Fauna triatomínica dos municípios do litoral norte do Estado de São Paulo, Brasil. Revista da Sociedade Brasileira de Medicina Tropical 36 (supl II): 60, 2003

12. Rodrigues VLCC, Silva EOR. Doença de Chagas caracterização da cepa isolada do primeiro caso humano autóctone do litoral sul do estado de São Paulo. In Resumos da VIII Reunião Anual de Pesquisa Básica em Doença de Chagas, Caxambu p. 40, 1981

13. Sherlock IA. Vetores. In: Brener Z, Andrade Z (eds) Trypanosoma cruzi e Doença de Chagas. Editora Guanabara-Koogan, Rio de Janeiro, p. 42-88, 1979.

14. Souza JMP, Silva EOR, Rodrigues VLCC. Triatoma sórdida-considerações sobre o tempo de vida das formas adultas e sobre a oviposição das fêmeas. Revista de Saúde Pública 12:291-296, 1978 